\title{
NEAR-ULTRAVIOLET EXCESS IN SLOWLY ACCRETING T TAURI STARS: LIMITS IMPOSED BY CHROMOSPHERIC EMISSION*
}

\author{
Laura Ingleby $^{1}$, Nuria Calvet ${ }^{1}$, Edwin Bergin ${ }^{1}$, Gregory Herczeg $^{2}$, AleXander Brown $^{3}$, Richard Alexander $^{4}$, \\ Suzan Edwards ${ }^{5}$, Catherine Espaillat ${ }^{6,15}$, Kevin France $^{3}$, Scott G. Gregory ${ }^{7}$, Lynne Hillenbrand ${ }^{7}$,

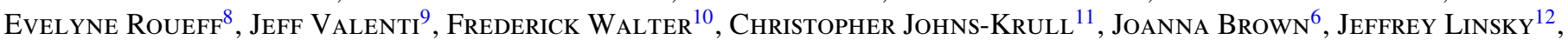 \\ Melissa McClure ${ }^{1}$, David Ardila ${ }^{13}$, Hervé Abgrall ${ }^{8}$, Thomas Bethell $^{1}$, Gaitee Hussain ${ }^{14}$, and Hao Yang ${ }^{12}$ \\ ${ }^{1}$ Department of Astronomy, University of Michigan, 830 Dennison Building, 500 Church Street, Ann Arbor, MI 48109, USA; \\ lingleby@umich.edu,ncalvet@umich.edu \\ 2 Max-Planck-Institut für Extraterrestriche Physik, Postfach 1312, D-85741 Garching, Germany; gregoryh@mpe.mpg.de \\ ${ }^{3}$ Center for Astrophysics and Space Astronomy, University of Colorado, Boulder, CO 80309-0389, USA \\ ${ }^{4}$ Department of Physics \& Astronomy, University of Leicester, University Road, Leicester LE1 7RH, UK \\ ${ }^{5}$ Department of Astronomy, Smith College, Northampton, MA 01063, USA \\ ${ }^{6}$ Harvard-Smithsonian Center for Astrophysics, 60 Garden Street, MS-78, Cambridge, MA 02138, USA \\ ${ }^{7}$ Department of Astrophysics, California Institute of Technology, MC 249-17, Pasadena, CA 91125, USA \\ ${ }^{8}$ LUTH and UMR 8102 du CNRS, Observatoire de Paris, Section de Meudon, Place J. Janssen, F-92195 Meudon, France \\ ${ }^{9}$ Space Telescope Science Institute, 3700 San Martin Drive, Baltimore, MD 21218, USA \\ ${ }^{10}$ Department of Physics and Astronomy, Stony Brook University, Stony Brook, NY 11794-3800, USA \\ ${ }^{11}$ Department of Physics and Astronomy, Rice University, Houston, TX 77005, USA \\ 12 JILA, University of Colorado and NIST, 440 UCB Boulder, CO 80309-0440, USA \\ ${ }^{13}$ NASA Herschel Science Center, California Institute of Technology, Mail Code 100-22, Pasadena, CA 91125, USA \\ ${ }^{14}$ ESO, Karl-Schwarzschild-Strasse 2, D-85748 Garching bei München, Germany \\ Received 2011 April 22; accepted 2011 October 27; published 2011 November 28
}

\begin{abstract}
Young stars surrounded by disks with very low mass accretion rates are likely in the final stages of inner disk evolution and therefore particularly interesting to study. We present ultraviolet (UV) observations of the $\sim 5-9 \mathrm{Myr}$ old stars RECX-1 and RECX-11, obtained with the Cosmic Origins Spectrograph and Space Telescope Imaging Spectrograph on the Hubble Space Telescope, as well as optical and near-infrared spectroscopic observations. The two stars have similar levels of near-UV emission, although spectroscopic evidence indicates that RECX-11 is accreting and RECX-1 is not. The line profiles of $\mathrm{H} \alpha$ and He I $\lambda 10830$ in RECX-11 show both broad and narrow redshifted absorption components that vary with time, revealing the complexity of the accretion flows. We show that accretion indicators commonly used to measure mass accretion rates, e.g., $U$-band excess luminosity or the $\mathrm{Ca}$ II triplet line luminosity, are unreliable for low accretors, at least in the middle $\mathrm{K}$ spectral range. Using RECX-1 as a template for the intrinsic level of photospheric and chromospheric emission, we determine an upper limit of $3 \times 10^{-10} M_{\odot} \mathrm{yr}^{-1}$ for RECX-11. At this low accretion rate, recent photoevaporation models predict that an inner hole should have developed in the disk. However, the spectral energy distribution of RECX-11 shows fluxes comparable to the median of Taurus in the near-infrared, indicating that substantial dust remains. Fluorescent $\mathrm{H}_{2}$ emission lines formed in the innermost disk are observed in RECX-11, showing that gas is present in the inner disk, along with the dust.
\end{abstract}

Key words: accretion, accretion disks - circumstellar matter - stars: pre-main sequence

Online-only material: color figures

\section{INTRODUCTION}

It is now accepted that classical T Tauri stars $\left(\mathrm{CTTS}^{16}\right)$ are accreting mass from their disks via magnetospheric accretion. In this process, the stellar magnetic field truncates the circumstellar disk and channels gas near the truncation radius onto the stellar surface where an accretion shock forms. Gas heated by the shock emits energetic photons which, after re-processing in the accretion streams, irradiate the inner disk with ultraviolet (UV) and X-ray photons (Calvet \& Gullbring 1998; Ardila \& JohnsKrull 2009; Günther et al. 2007). This energetic emission plays a key role in driving disk chemistry and may be the most efficient mechanism for dispersing the disk gas (Najita et al. 2010; Pinte

\footnotetext{
* This paper includes data gathered with the $6.5 \mathrm{~m}$ Magellan Telescopes located at Las Campanas Observatory, Chile.

15 NSF Astronomy and Astrophysics Postdoctoral Fellow.

16 In this paper, we use the terms classical and weak-line T Tauri stars (CTTS and WTTS) to indicate accreting and non-accreting T Tauri stars, respectively, following White \& Basri (2003).
}

et al. 2010; Bethell \& Bergin 2009; Gorti et al. 2009). The rate at which material is accreted is an important input in models of circumstellar disks, affecting the surface density and inner disk heating (D'Alessio et al. 2006), so accurate accretion rates are essential for describing the evolution of both gas and dust. Accretion rates decrease with age (Calvet et al. 2005), so objects with very low accretion rates are particularly interesting for studying the final stages in the evolution of disks.

The spectrum produced by the accretion shock on the stellar surface peaks in the UV, so UV flux excesses over the stellar fluxes are the most direct measure of the accretion luminosity $\left(L_{\text {acc }}\right)$ from which the mass accretion rate, $\dot{M}$, can be derived (Section 4.2). However, CTTS have active chromospheres which produce UV emission in excess of the main-sequence standards (Houdebine et al. 1996; Franchini et al. 1998). The chromospheric contribution to the measured excess is expected to be small when compared to the excess due to accretion in CTTS with typical values of the mass accretion rate $\dot{M} \sim 10^{-8} M_{\odot} \mathrm{yr}^{-1}$ (Gullbring et al. 1998). However, 
as the disk evolves, the mass accretion rate and accretion luminosity decrease, and so do the accretion-powered UV fluxes. In contrast, indicators of chromospheric activity, e.g., the $\mathrm{X}$-ray luminosity, stay approximately constant in the age range 1-10 Myr during which disks evolve significantly (Ingleby et al. 2011). Therefore, the chromospheric contribution is expected to become more important in determining the total UV flux as the disk evolves and at some point will become the dominant contributor. From this point on, the UV excess is no longer measuring accretion.

In most determinations of accretion luminosity in CTTS to date, the excess fluxes in the UV have been measured using main-sequence standards as templates for the intrinsic stellar emission. A better template for the stellar emission of an accreting T Tauri star is a non-accreting T Tauri star (or WTTS), which is expected to have a comparable level of chromospheric activity as a CTTS. However, UV observations of WTTS with enough sensitivity do not exist (Valenti et al. 2003), so it has not been possible to determine typical levels of chromospheric emission, which in turn are important to set up realistic limits to the accretion luminosities that can be determined from UV observations. In this paper, we address these issues by comparing the UV fluxes of a WTTS, RECX-1, with those of a very low accretor, RECX-11, and find that the NUV fluxes are dominated by chromospheric emission; this allows us to place limits on the lowest accretion luminosities detectable in the UV.

Even for chromospheric-dominated UV fluxes, alternative indicators may be used to identify whether a young star is accreting. A commonly used accretion indicator is the $\mathrm{H} \alpha$ line profile. $\mathrm{H} \alpha$ is observed in emission in all T Tauri stars but a large velocity width or line asymmetry indicates accretion, whereas a narrow symmetric line profile is characteristic of a non-accreting star (Muzerolle et al. 2001; Lima et al. 2010; White \& Basri 2003). In addition, redshifted absorption components superimposed on the emission line profiles are direct indication of infalling material and can be explained in terms of magnetospheric accretion (Muzerolle et al. 1998, 2001). The He I line at $10830 \AA$ can also be used to diagnose accretion properties in T Tauri stars. In CTTS this line shows deep blueshifted and redshifted absorption components in its profile, which have been interpreted as formed in outflows and accretion streams (Edwards et al. 2006; Fischer et al. 2008). Other indicators of accretion used in the literature are the forbidden lines of [O I] 6300 and $6363 \AA$, which are present only in accreting stars (Hartigan et al. 1995), and the $\mathrm{Ca}$ II triplet lines, whose luminosities correlate with accretion luminosity (Calvet et al. 2004). In this paper, we analyze these accretion indicators in high-resolution spectra of RECX-11 and RECX-1. We find conclusive evidence for accretion in RECX-11 and not in RECX-1 in our high-resolution observations of $\mathrm{H} \alpha$ and $\mathrm{He}$ I $\lambda 10830$, but not in other lines. Our analysis allows us to assess which accretion indicators are appropriate for detecting accretion at very low levels.

The indicators discussed above are directly tracing material in the accretion shock or flows, but another indirect method of determining whether a source is accreting or not involves gas in the inner circumstellar disk. While in the past circumstellar gas was difficult to observe, new observations have made gas detections possible (Bary et al. 2008; Bitner et al. 2008; Carr \& Najita 2008; Pascucci et al. 2009). Of particular interest are observations of hot gas very close to the star, near the magnetospheric truncation radius. FUV observations have shown that $\mathrm{CO}$ and $\mathrm{H}_{2}$ at temperatures near $2500 \mathrm{~K}$ are present in the disk (France et al. 2010, 2011; Herczeg et al. 2002, 2004).
Ingleby et al. (2009) analyzed a sample of FUV spectra of $\mathrm{T}$ Tauri stars and found that $\mathrm{H}_{2}$ was present in the inner $1 \mathrm{AU}$ of all accreting sources but not in non-accreting sources. FUV gas detections therefore indicate more than the presence of gas, but actually show whether a source is still accreting or not. These results were based on Advanced Camera for Surveys low-resolution observations; the Cosmic Origins Spectrograph (COS) observations presented in this paper allow us to confirm this finding using spectrally resolved $\mathrm{H}_{2}$ lines.

This paper is organized as follows. In Section 2, we present the UV, optical, and IR data discussed in this paper. In Section 3, we discuss which commonly used accretion indicators are valid at low levels of accretion and which are unreliable. In Section 4, we estimate limits for the minimum $\dot{M}$ that can be determined from the UV. Finally, in Section 5 we discuss the limitations of current accretion rate determinations in view of our results, as well as the implications for disk evolution models.

\section{OBSERVATIONS}

\subsection{The Targets}

Our targets are the young stars RECX-1 and RECX-11 in the $\eta$ Chamaeleontis group, the nearest open cluster at only 97 pc with an age of 5-9 Myr (Mamajek et al. 1999, 2000; Luhman \& Steeghs 2004). With $A_{V} \sim 0$, this region has little extinction (Luhman \& Steeghs 2004), making it ideal for UV observations as corrections for reddening may introduce large uncertainties. The sources have similar spectral types, and were classified as K4 by Mamajek et al. (1999) and K5-K6 by Luhman \& Steeghs (2004). Sources in this region have also been well characterized in the infrared with broadband $J-L$ photometry (Lyo et al. 2004), Spitzer Infrared Array Camera (IRAC) and Multiband Imaging Photometer (MIPS) photometry, and Infrared Spectrograph (IRS) spectroscopy (Sicilia-Aguilar et al. 2009). The properties of each source are summarized in Table 1.

\subsection{HST Observations}

Observations of RECX-1 and RECX-11 were obtained between 2009 December and 2010 January with COS and Space Telescope Imaging Spectrograph (STIS) on Hubble Space Telescope (HST) in GO Program 11616 (PI: Herczeg). STIS NUV observations used the MAMA detector and the G230L grating providing spectral coverage from 1570 to $3180 \AA$ with $R \sim 500-1000$. Optical observations were completed during the same orbit as the NUV using the G430L grating which covers 2900-5700 $\AA$ with $R \sim 530-1040$, resulting in almost simultaneous NUV to optical coverage with STIS.

The low-resolution STIS spectra were calibrated with customwritten IDL routines following the procedures described in the STIS data handbook. The wavelengths were calibrated from the location of identified emission lines within the spectrum, and fluxes were calibrated from spectra of WD $1337+705$ in the NUV and HIP 45880 in the optical. The flux calibration also includes a wavelength-dependent aperture correction.

COS observations of RECX-11 were presented in France et al. (2011), and we discuss them here together with observations for RECX-1. The COS FUV observations were taken with the G160M and G130M gratings and were completed within 3-4 hr of the STIS observations. The combination of the two gratings provides FUV coverage from 1150 to $1775 \AA$ with $R \sim 16,000-18,000$. The $\log$ of the observations is given in Table 2 . 
Table 1

Properties of RECX-1, RECX-11, and HD 154363

\begin{tabular}{lccccccc}
\hline \hline Object & Class & Spectral Type & $\begin{array}{c}\text { Luminosity } \\
\left(L_{\odot}\right)\end{array}$ & $\begin{array}{c}\text { Mass } \\
\left(M_{\odot}\right)\end{array}$ & $\begin{array}{c}\text { Radius } \\
\left(R_{\odot}\right)\end{array}$ & $\begin{array}{c}\text { Distance } \\
(\mathrm{pc})\end{array}$ & $\begin{array}{c}\text { Age } \\
(\mathrm{Myr})\end{array}$ \\
\hline RECX-1 & WTTS & K5-K6 & 1.0 & 0.9 & 1.8 & 97 & $5-9$ \\
RECX-11 & CTTS & K5-K6 & 0.6 & 1.0 & 1.4 & 97 & $5-9$ \\
HD 154363 & Dwarf & K5 & 0.2 & 0.6 & 0.8 & 11 & $<1400$ \\
\hline
\end{tabular}

Notes. Masses for the $\eta$ Cha sources were calculated from the position in the H-R diagram, assuming the evolutionary tracks of Siess et al. (2000) with solar metallicity. Stellar parameters for HD154363 are from Holmberg et al. (2009) and Takeda et al. (2007).

Table 2

Log of Observations

\begin{tabular}{|c|c|c|c|c|}
\hline Object & $\begin{array}{l}\text { R.A. } \\
\text { (J2000) }\end{array}$ & $\begin{array}{l}\text { Decl. } \\
\text { (J2000) }\end{array}$ & Telescope/Instrument & Date of Observation \\
\hline \multirow[t]{4}{*}{ RECX-1 } & 083656.12 & -785645.3 & $H S T / \mathrm{COS}$ G130M/G160M & 2010 Jan 22 \\
\hline & & & $H S T /$ STIS G230L/G430L & 2010 Jan 22 \\
\hline & & & Magellan/MIKE & 2010 Mar 10 \\
\hline & & & VLT-UT1/CRIRES & 2011 May 22 \\
\hline \multirow[t]{9}{*}{ RECX-11 } & 084701.28 & -785934.1 & HST/COS G130M/G160M & 2009 Dec 12 \\
\hline & & & $H S T / \mathrm{STIS}$ G230L/G430L & 2009 Dec 12 \\
\hline & & & Magellan/MIKE & 2010 Mar 10 \\
\hline & & & CTIO/SMARTS RC Spectrograph & 2009 Nov 26 \\
\hline & & & CTIO/SMARTS RC Spectrograph & 2009 Nov 27 \\
\hline & & & CTIO/SMARTS RC Spectrograph & 2009 Dec 15 \\
\hline & & & CTIO/SMARTS RC Spectrograph & 2009 Dec 19 \\
\hline & & & Gemini South/Phoenix & 2009 Dec 12 \\
\hline & & & VLT-UT1/CRIRES & 2011 May 22 \\
\hline
\end{tabular}

The COS spectra were processed by the standard CALCOS calibration pipeline. The individual spectral segments, after obtaining different wavelength settings to minimize instrumental fixed pattern effects and to provide full wavelength sampling, were combined using the IDL co-addition procedure described by Danforth et al. (2010). This procedure co-aligns the individual exposures and performs an exposure-weighted interpolation onto a common wavelength grid.

In our COS NUV acquisition image, we resolved RECX-1 into a binary with a separation of 0.141 and a position angle of $21^{\circ}$ (taking into account the fact that MIRRORB creates spurious images), indicating some relative motion between the 2004 April $H$-band images of Brandeker et al. (2006) and our 2010 January observations. Brandeker et al. (2006) found that the semi-major axis of the orbit was 0 "'42. The two stars are also marginally resolved in our STIS spectra, which were obtained with a slit position angle of -49.6 . Separate spectral extractions for the components were obtained by fitting two Gaussians to the crossdispersed profile at each wavelength position, subtracting off the emission from one component, and subsequently extracting the counts in the other component. From the blue spectrum, we estimate spectral types of $\mathrm{K} 4$ for the $\mathrm{S}$ component and $\mathrm{K} 6$ for the $\mathrm{N}$ component, consistent with the unresolved spectral type of K5-K6 (Luhman \& Steeghs 2004). The near-UV spectra of the two stars are similar, except for a $\sim 1.5$ times stronger $\mathrm{Mg}$ II $\lambda 2800$ flux from the S component. For our analysis, we use the combined spectra of the $\mathrm{N}$ and $\mathrm{S}$ components to increase the signal to noise. With both components having spectral types close to RECX-11 and very similar STIS spectra, little error is introduced by using the combined spectra.

\subsection{Ground-based Observations}

Both sources were observed using Magellan Inamori Kyocera Echelle (MIKE) on the Magellan-Clay telescope at Las
Campanas Observatory in Chile (Bernstein et al. 2003) on 2010 March 11, with a coverage of 4800-9000 $\AA$ and resolution $R \sim 35,000$. The data were reduced using the Image Reduction and Analysis Facility (IRAF) tasks CCDPROC, APFLATTEN, and DOECSLIT (Tody 1993).

We also obtained four low-dispersion spectra of RECX-11, covering the $\mathrm{H} \alpha$ line, between 2009 November 27 and December 22, using the Small and Medium Aperture Research Telescope System (SMARTS) $1.5 \mathrm{~m}$ telescope at CTIO. We used the RC spectrograph, which is a long-slit spectrograph with a Loral CCD detector; the slit subtends 5 arcmin on the sky and has a 1.5 arcsec slit width. All observations were made by SMARTS service observers and each night consisted of three observations with 5 minute integration time. The three observations are median filtered to minimize contamination by cosmic rays. The data were reduced with the SMARTS spectroscopic data reduction pipeline. ${ }^{17}$ We subtracted the bias and trimmed the overscan and flattened the image using dome flats. The spectra were extracted by fitting a Gaussian plus a linear background at each column. Uncertainties were based on counting statistics, including uncertainties in the fit background level. The wavelength calibration was based on an arc lamp spectrum obtained before each set of images.

Finally, we obtained $R=100,000$ spectra of the He I $\lambda 10830$ line from RECX-11 with Phoenix on Gemini South on 2009 December 12 and from both RECX-1 and RECX-11 using CRIRES on VLT-UT1 on 2011 May 22. The observations were obtained in an ABBA nod pattern. The star $\eta$ Cha, a B8 premain-sequence star with no detectable photospheric features at $10830 \AA$, was observed to provide the telluric correction. These spectra were reduced with custom-written routines in IDL. The

\footnotetext{
17 http://www.astro.sunysb.edu/fwalter/SMARTS/smarts_15ms ched.html\#RCpipeline
} 


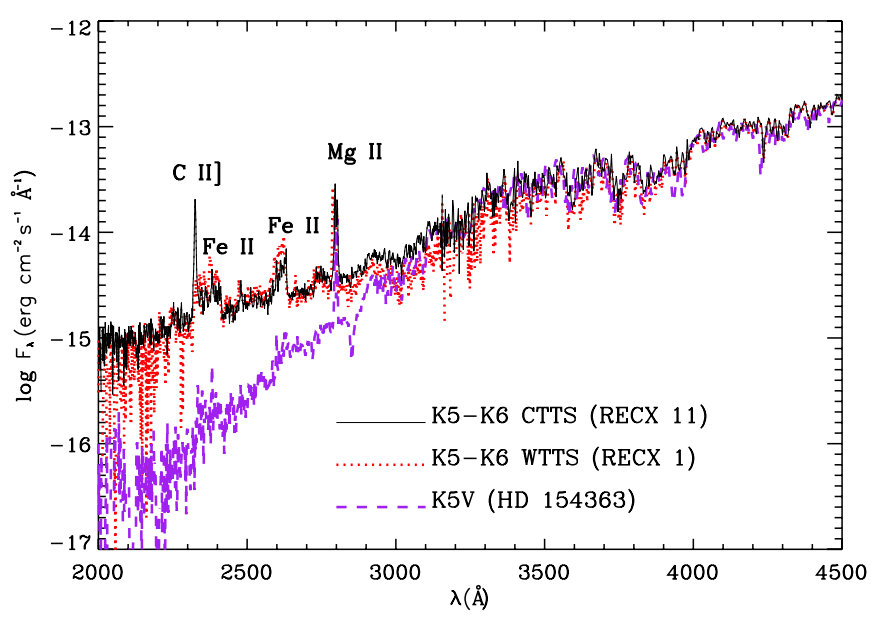

Figure 1. STIS spectra of the WTTS RECX-1 (both north and south components, see Section 2.2), the CTTS RECX-11, and the dwarf standard star, HD 154363. All have K5-K6 spectral types but RECX-1 is no longer accreting while RECX-11 has ongoing accretion (Lawson et al. 2004; Sicilia-Aguilar et al. 2009; Jayawardhana et al. 2006). The spectra of RECX-1 and RECX-11 are remarkably similar, except for the stronger C II $\lambda 2325$ line in the CTTS. The Mg II $\lambda 2800$ doublet strength is comparable in the three sources (Section 4).

(A color version of this figure is available in the online journal.)

wavelength solution is accurate to $\sim 1 \mathrm{~km} \mathrm{~s}^{-1}$ and was calculated from a linear fit to telluric absorption lines obtained from spectra generated by ATRAN (Lord 1992) and available online.

\section{ACCRETION ANALYSIS}

Figure 1 shows the STIS NUV spectra of RECX-1 and RECX-11; they are remarkably similar, making it difficult to assess if either is accreting based on a UV excess. Also shown in Figure 1 is a STIS spectrum of the inactive $\mathrm{K} 5 \mathrm{~V}$ star HD 154363 (Martínez-Arnáiz et al. 2010; properties in Table 1) taken from the HST/STIS Next Generation Spectral Library (Heap \& Lindler 2007), for comparison. Both $\eta$ Cha sources have excess NUV emission compared to the mainsequence standard (Section 4.1). Despite the similarity of NUV fluxes, analysis of emission line profiles shows that one source, RECX-11, is still accreting material from the disk while the other, RECX-1, is not. In this section we review this evidence. We find that the emission line profiles of RECX-11 show redshifted absorption components indicative of accreting material, and consistent with magnetospheric accretion. As mentioned before, accretion shock emission is then expected from the accreting star, but it must be hidden by intrinsic chromospheric emission present in both the accreting and the non-accreting stars; this points to a very low accretion luminosity and mass accretion rate. In Section 4 we estimate upper limits for these quantities.

\subsection{Diagnostics of Low Accretion}

\subsubsection{Lines Produced by Accretion and Related Processes}

Our spectroscopic observations, from the FUV to the IR, include a number of emission lines that trace phenomena such as accretion and outflows in T Tauri stars. Line widths of several hundred $\mathrm{km} \mathrm{s}^{-1}$ are used to identify accretors, but the most conspicuous indicators are redshifted and blueshifted absorption components on the emission line profiles.

Accessible in the optical, the $\mathrm{H} \alpha$ emission line is one of the most commonly used accretion tracers. Figure 2 shows

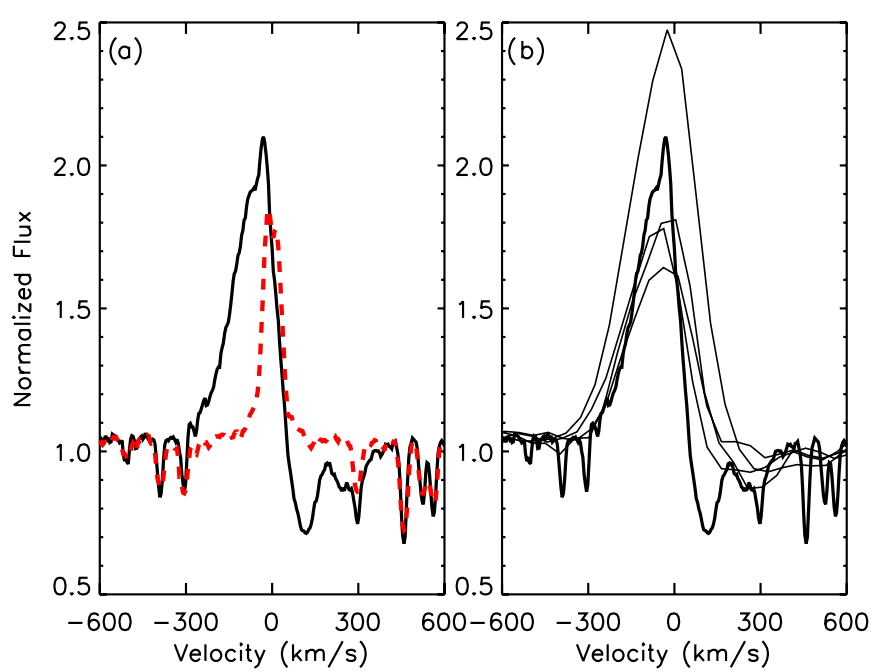

Figure 2. H $\alpha$ line profiles. (a) MIKE spectra of RECX-11 (solid line) and RECX1 (red dashed line). (b) SMARTS spectra of RECX-11 obtained within a few days of the HST observations. The MIKE spectrum is also shown for comparison as the thick solid line. The wide blue wing and redshifted absorption in the $\mathrm{H} \alpha$ profile of RECX-11, characteristic of accretion, are observed in the MIKE and SMARTS data. Transient narrow redshifted components are observed in the red wing of the lines and show the largest variability.

(A color version of this figure is available in the online journal.)

MIKE $\mathrm{H} \alpha$ line profiles for RECX-11 and RECX-1. The $\mathrm{H} \alpha$ equivalent widths (EWs) of RECX-11 and RECX-1 are $4 \AA$ and $1.3 \AA$, respectively, which would make RECX-1 a WTTS and RECX-11 a borderline CTTS/WTTS according to the standard criterion for a K5-K6 star (White \& Basri 2003). The $\mathrm{H} \alpha$ profile of RECX-1 in Figure 2(a) agrees with the WTTS classification because it is symmetric and narrow. With a line width at $10 \%$ of the peak intensity of only $\sim 130 \mathrm{~km} \mathrm{~s}^{-1}$, the line shows no indication of the high velocities that are characteristic of magnetospheric accretion. In contrast, the $\mathrm{H} \alpha$ profile of RECX-11 is typical of accreting sources, with a line width at $10 \%$ of $\sim 300 \mathrm{~km} \mathrm{~s}^{-1}$ (White \& Basri 2003). The blue emission wing is wide, extending to velocities of several hundred $\mathrm{km} \mathrm{s}^{-1}$, implying that material is accreting at nearly free-fall velocities. Inverse P Cygni absorption due to infalling material along the accretion streams is also seen (Lima et al. 2010; Muzerolle et al. 2003; Walter 1999). The line profile is consistent with model predictions for a high-inclination source (Muzerolle et al. 2001; Kurosawa et al. 2006), in agreement with the inclination of $i \sim 68^{\circ}$ estimated from the values of the projected rotation velocity $v \sin i$ (Jayawardhana et al. 2006) and the rotational period (Lawson et al. 2001). Moreover, Lawson et al. (2004) compared their observed $\mathrm{H} \alpha$ profile of RECX-11 with magnetospheric accretion models and obtained a good fit assuming an inclination of $i \sim 70^{\circ}$.

In Figure 2(b), we show our MIKE profile and the four SMARTS low-resolution $\mathrm{H} \alpha$ profiles of RECX-11 obtained within a few days before and after the HST observations. The $\mathrm{H} \alpha$ profile is highly variable, as already noted by Lawson et al. (2004) and Jayawardhana et al. (2006). The wide blue emission wing is present in all spectra, but variability is conspicuous in the red wing, sometimes showing narrow transient redshifted absorption components. This variability points to a complex geometry of the accretion streams very close to the star since redshifted absorption occurs when material in the accretion columns absorbs hot radiation produced in the accretion shock on the stellar surface (Bouvier et al. 2007; Muzerolle et al. 


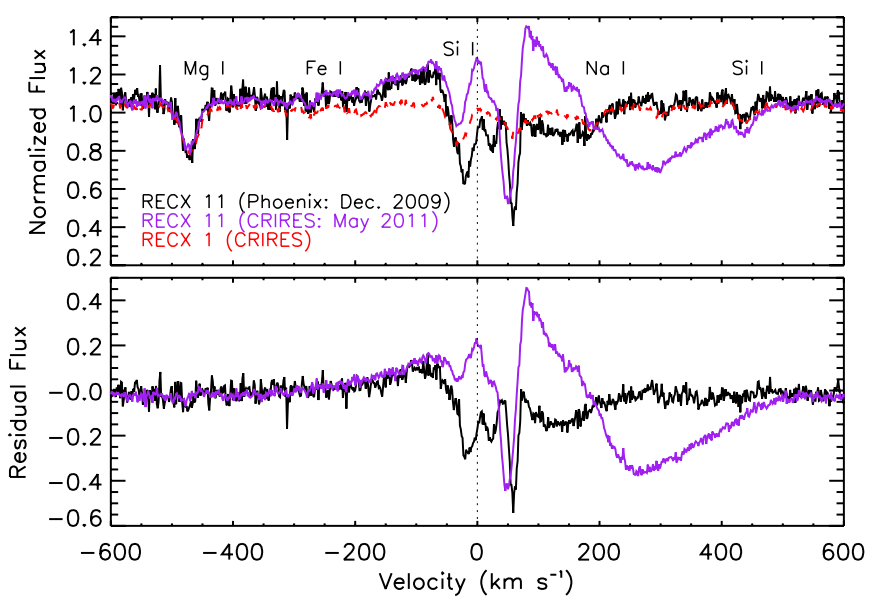

Figure 3. He I $\lambda 10830$ profiles of RECX-1 and RECX-11. Top panel: He I observations of RECX-11 are shown from two epochs largely separated in time by 18 months. RECX-1 is shown for comparison and photospheric lines identified by the Infrared Telescope Facility (IRTF) spectral library are designated (Rayner et al. 2009). RECX-11 shows significant variability between the two observations, including a variable redshifted emission component and a broad, high-velocity redshifted absorption component, characteristic of magnetospheric infall. Bottom panel: He I $\lambda 10830$ line profiles of RECX-11 after subtraction of the RECX-1 line profile. The Phoenix observation was degraded to the resolution of the CRIRES observations prior to subtraction of the RECX-1 profile. The photospheric lines are cleanly subtracted but the complex nature of the profile remains.

(A color version of this figure is available in the online journal.)

2001). The complex structure revealed by the line profiles of $\mathrm{H} \alpha$, which in the case of RECX-11 is conclusive in identifying accretion, would have been missed when only considering the equivalent width of the line.

In Figure 3, we show two He I $\lambda 10830$ profiles for RECX11 obtained 18 months apart with Phoenix and CRIRES along with a CRIRES spectrum of RECX-1 for comparison. We also show the RECX-11 line profiles after subtraction of the RECX1 line profile (with the Phoenix observation at the resolution of CRIRES) which removes any contribution by photospheric absorption lines. The He I $\lambda 10830$ emission profiles show absorption components similar to those found by Edwards et al. (2006) and Fischer et al. (2008) in a large sample of CTTS, which were interpreted as arising in the wind and in the accretion columns. The earlier Phoenix profile shows a broad redshifted absorption component extending to $\sim 250 \mathrm{~km} \mathrm{~s}^{-1}$ with several deep, narrow redshifted and blueshifted absorption lines at lower velocities, between -50 and $100 \mathrm{~km} \mathrm{~s}^{-1}$. The second, CRIRES, profile also shows a complex absorption spectrum with a broad component extending from 200 to $400 \mathrm{~km} \mathrm{~s}^{-1}$. It also shows a redshifted emission wing which was not observed in the Phoenix observation. The broad highvelocity absorption feature in the CRIRES spectrum occurs at velocities typical of material infalling along accretion streams. According to magnetospheric accretion models, material falling from $5 R_{*}$ in the disk onto a star with the mass and radius of RECX-11 will reach velocities of $\sim 470 \mathrm{~km} \mathrm{~s}^{-1}$, capable of producing the absorption features at the observed velocities (Calvet \& Gullbring 1998).

Fischer et al. (2008) found that sources with low accretion rates, observed to have low veiling at $1 \mu \mathrm{m}\left(R_{Y}<0.5\right)$, were more likely to have redshifted absorption in He I than sources with high accretion rates. Fischer et al. (2008) also found that in order to explain the He I $\lambda 10830$ profiles absorption over a large range of velocities was necessary. They proposed

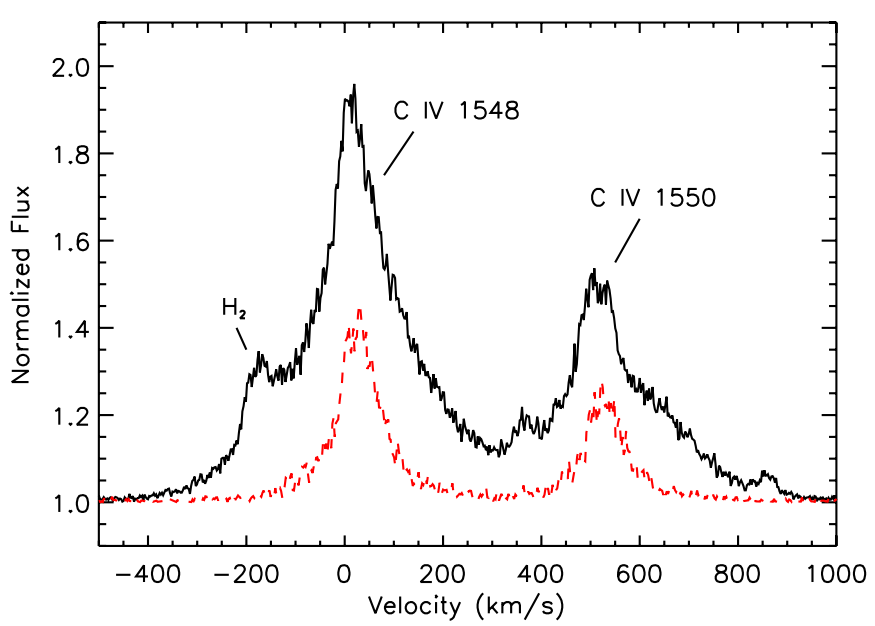

Figure 4. COS C IV $\lambda 1549$ and $\lambda 1551$ line profiles for RECX-11 (solid line) and RECX-1 (red dashed line). In agreement with other accretion indicators, the CTTS RECX-11 has broader lines than the WTTS RECX-1; the emission is expected to arise in the accretion shock and flows.

(A color version of this figure is available in the online journal.)

that in low $\dot{M}$ sources accretion occurs in low-density, narrow accretion "streamlets" with small filling factors, covering a large velocity range, although none of their sources had low enough accretion rates to test this scenario. The narrow redshifted absorption features in the Phoenix spectrum of our very low accretor, RECX-11 (Section 4), may be observational evidence for these multiple, narrow accretion streams. Some of these narrow features, like the one at $\sim 100 \mathrm{~km} \mathrm{~s}^{-1}$, may be related to the feature at comparable velocity seen in the MIKE H $\alpha$ profile (Figure 2), although no definite conclusion can be stated since the lines were observed at different times. Simultaneous extensive coverage of $\mathrm{H} \alpha$ and $\mathrm{He}$ I $\lambda 10830$ is necessary to understand the complexity of the accretion flows in RECX-11.

In addition to $\mathrm{H} \alpha$ and $\mathrm{He}_{\mathrm{I}} \lambda 10830$, the $\mathrm{C}$ IV doublet in the FUV spectrum can be used to separate accretors from nonaccretors. The C IV $\lambda 1549$ line emission has been shown to correlate with the mass accretion rate, indicating that its origin is, at least in part, in the accretion shock and flows (Johns-Krull et al. 2000; Calvet et al. 2004). In Figure 4, we compare the $\mathrm{C}$ IV line profiles of the accretor, RECX-11, to the non-accretor, RECX-1. The C IV line profile is much wider and stronger in the accreting source than in the WTTS in which the emission is expected to be chromospheric. The strength and width of C IV in RECX-11 are consistent with the accreting nature of RECX-11 (D. Ardila et al., in preparation).

In the NUV range, the only significant difference between the spectra of RECX-11 and RECX-1 is in the [C II] $\lambda 2325$ line, which is much stronger in RECX-11 (Figure 1). This line has been observed in other accreting sources (Gómez de Castro \& Ferro-Fontán 2005; Calvet et al. 2004), and its likely origin is the accretion shock.

\subsubsection{The $\mathrm{H}_{2}$ Lines}

The FUV spectrum of RECX-11 contains a wealth of lines due to molecular species, including $\mathrm{H}_{2}$ (France et al. 2011). Lines from $\mathrm{H}_{2}$ in the UV are mainly due to two mechanisms. Ly $\alpha$ emission excites $\mathrm{H}_{2}$, producing a fluorescent spectrum in the UV as the electrons cascade back to the ground electronic state (Herczeg et al. 2002, 2004; Yang et al. 2011). In addition, fast electrons can collisionally excite $\mathrm{H}_{2}$, resulting in UV lines and continua (Bergin et al. 2004; Ingleby et al. 2009). 


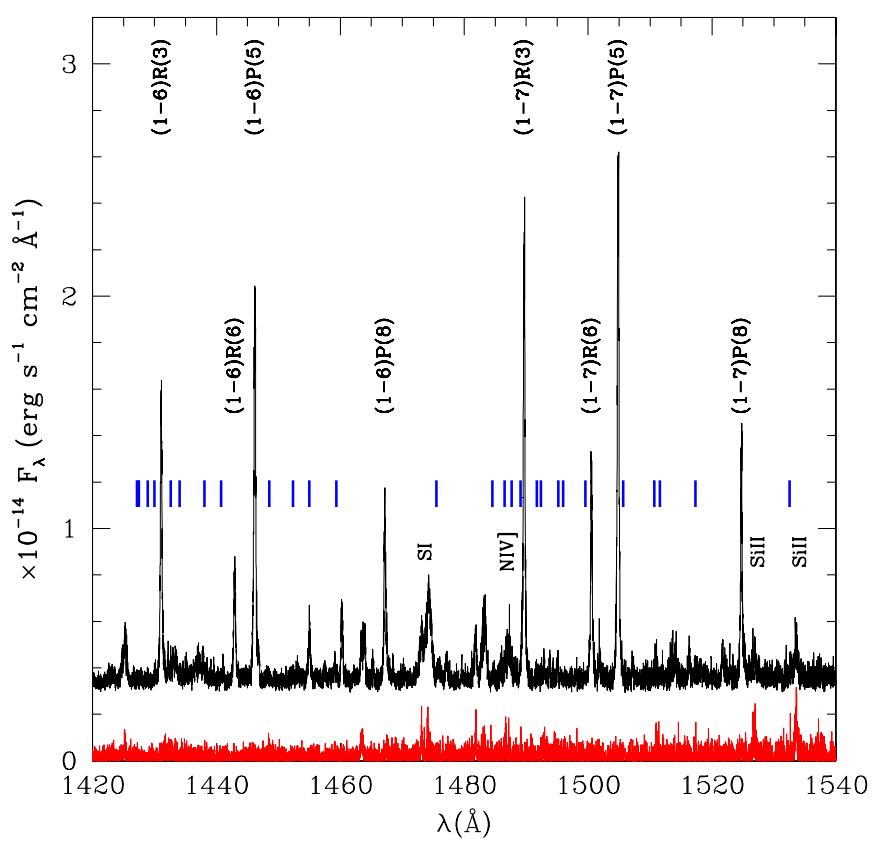

Figure 5. COS FUV spectra of the WTTS RECX-1 (lower red) and the CTTS RECX-11 (upper black). The RECX-11 spectrum is offset vertically for clarity. Strong lines of $\mathrm{Ly} \alpha$-fluoresced $\mathrm{H}_{2}$ and atomic lines are labeled. The blue dashes show the wavelengths at which additional $\mathrm{H}_{2}$ emission lines from $\mathrm{Ly} \alpha$ pumping may be seen. RECX-1 shows a lack of molecular gas lines which are apparent in RECX-11; essentially no gas exists in the inner disk region of RECX-1.

(A color version of this figure is available in the online journal.)

Using emission from collisionally excited $\mathrm{H}_{2}$ measured in low-resolution FUV spectra of a large sample of both accreting and non-accreting young stars, Ingleby et al. (2009) showed that by the time accretion ends, the inner disks are depleted of gas. Our targets confirm this trend at high resolution. In Figure 5, we show the COS FUV spectrum of RECX-11 compared to RECX-1; the wavelengths at which $\mathrm{H}_{2}$ pumped by Ly $\alpha$ may emit are labeled. $\mathrm{H}_{2}$ emission lines at 1434.1 and $1445.3 \AA$ may also be pumped by the $\mathrm{C}$ III $\lambda 1174$ multiplet (Herczeg et al. 2002). Figure 5 shows that the COS FUV spectrum of RECX-11 is rich in lines of molecular gas, similar to previously observed sources with confirmed accretion (Bergin et al. 2004; Herczeg et al. 2002, 2004; France et al. 2010). In contrast, molecular lines are not apparent in the FUV spectra of the WTTS RECX-1. If present, the flux of the $\mathrm{H}_{2}$ lines in RECX-1 is $<5 \%$ of the $\mathrm{H}_{2}$ flux in RECX-11.

Assuming that the molecular lines are formed in the atmosphere of the disk (Herczeg et al. 2004), we constructed synthetic models for an optically thin line formed between radii $R_{1}$ and $R_{2}$ in a Keplerian disk. We assumed that the emissivity depended on radius as $R^{\gamma}$ and that the intrinsic line profile was a Gaussian with width corresponding to a temperature of $2500 \mathrm{~K}$. The intensity at each radius was convolved with the rotational profile corresponding to the Keplerian velocity of the annulus, and the flux was then calculated by integrating the intensity over the range of radii. Finally, the resultant profile was convolved with the G130M line-spread function.

Figure 6 compares the predicted profile to a flux-weighted average of $\mathrm{H}_{2}$ lines, selected from the spectral region $(<1330 \AA)$ in which all the lines were observed with the same grating, G130M. The continuum of the predicted profile has been scaled to the observed continuum. The shaded region in Figure 6 corresponds to predicted profiles for models with inner radius

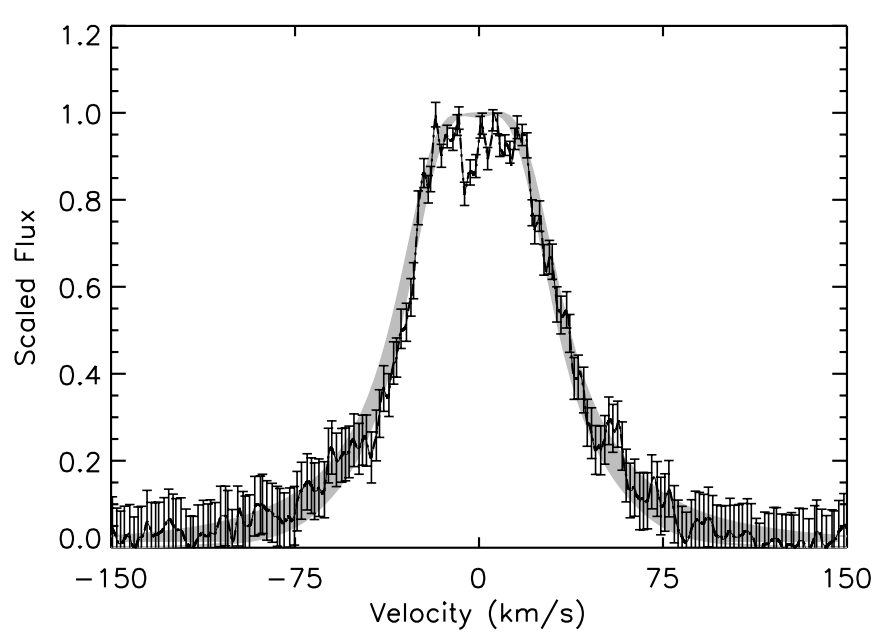

Figure 6. Spectrally resolved $\mathrm{H}_{2}$ line profile. The dot-dashed line shows the flux-weighted average of four $\mathrm{H}_{2}$ lines observed with the COS G130M grating. These lines result from the (1-2) $\mathrm{R}(6)$ and (1-2) $\mathrm{P}(5)$ Ly $\alpha$ pumping transitions and are found in a narrow wavelength region observed with a single grating. The shaded region shows the predicted line emission originating in a Keplerian disk with inclination of $68^{\circ}$, inner radius $R_{1}=1-20 R_{*}$, and outer radius $R_{2}=450-500 R_{*}$. The predicted line fluxes are convolved with the COS line-spread function to account for instrumental effects.

between $R_{1}=1-20 \quad R_{*}(0.01-0.1 \mathrm{AU})$, outer radius $R_{2}=$ 50-500 $R_{*}(0.3-3.2 \mathrm{AU})$, and emissivity power-law exponent $\gamma=-1.5 . \chi_{\text {red }}^{2}=3.1-3.6$ for the range of models shown in Figure 6 . We find that the inner radius is well constrained, within the uncertainties of the observations; small inner radii with high Keplerian velocities are needed to account for the width of the observed wings. The outer radius $R_{2}$, on the other hand, is not well constrained, since little emission comes from those regions. The power law of the emissivity is constrained by the width of the line near the peak, and we require it to fall rapidly with radius. This rapid falloff is likely due to the combination of a number of factors. For instance, the excitation will decrease as the flux of Ly $\alpha$ decreases with distance; similarly, the number of electrons in excited states that can absorb Ly $\alpha$ transitions decreases with radius, as the temperature of the upper disk layers drops (Meijerink et al. 2008). Our modeling indicates that the region of formation of the $\mathrm{H}_{2}$ lines can be as close to the star as 0.01-0.1 AU, as was also found for the accreting brown dwarf 2M1207 (France et al. 2010). This indicates that gas likely extends close to the stellar surface, inside the dust truncation radius, and reaches the stellar magnetosphere (see Section 5.2).

\subsection{Unreliable Accretion Diagnostics at Low $\dot{M}$}

Additional tracers of accretion present in our data set, such as [O I] at 6300 and $6363 \AA$ and the Ca II infrared triplet, do not provide conclusive indication of accretion in RECX-11. [O I] emission, which is expected to originate in an outflow, is observed only in sources undergoing accretion (Hartigan et al. 1995). We show in Figure 7(a) the line profiles of [O I] $\lambda 6300$ of RECX-11 and RECX-1. [O I] $\lambda 6300$ appears in RECX-11 in excess over the WTTS spectrum of RECX-1, but the detection is $<3 \sigma$, and therefore not reliable. Similarly, the Ca II $\lambda 8542$ is often used as an indicator of accretion, and even used to measure mass accretion rates (Muzerolle et al. 1998). However, the profile of this line in RECX-11, shown in Figure 7(b), is indistinguishable from the same line in RECX-1, indicating that the flux comes mostly from the chromosphere. 


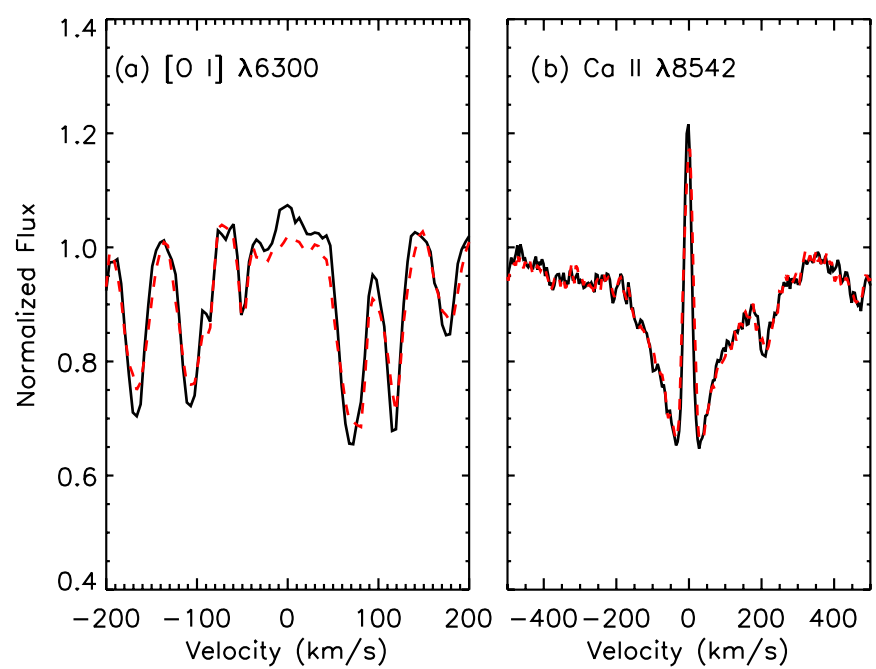

Figure 7. Accretion indicators. In each panel the black solid line shows the line profile of the CTTS, RECX-11, and the red dashed line shows the WTTS, RECX-1. (a) [O I]. The [O I] $\lambda 6300$ line probes wind emission in T Tauri stars. Weak [OI] emission appears to be present in RECX-11 in excess over the WTTS but the detection is $<3 \sigma$. If [OI] were present, it would be additional support for accretion in RECX-11. (b) Ca II. The Ca II $\lambda 8542$ shows an emission core on the photospheric absorption line. At low $\dot{M}$, this line is dominated by chromospheric emission and cannot be used to measure accretion rates, as shown by the identical profiles of RECX-1 and RECX-11.

(A color version of this figure is available in the online journal.)

The $\mathrm{Mg}$ II $\lambda 2800$ line luminosity also correlates with accretion luminosity in accreting stars (Calvet et al. 2004) and is seen in our STIS spectra (Figure 1). The line is observed to have the same strength in the weakly accreting star, the WTTS, and the dwarf star, indicating that it is mainly due to chromospheric activity. Moreover, its strength does not increase with the NUV emission level of the chromosphere. This agrees with the results of Cardini \& Cassatella (2007), who show that at ages $<0.3 \mathrm{Gyr}$, the $\mathrm{Mg}$ II $\lambda 2800$ fluxes are not observed to change due to saturation effects, and so the line strength does not reflect the level of chromospheric activity.

\section{MEASURING SMALL ACCRETION RATES}

\subsection{The Chromosphere}

While dwarf or giant stars have been used to estimate the intrinsic stellar emission in CTTS, when measuring the UV excess WTTS are the best templates for accretion analysis because they have similar surface densities and are expected to have comparable levels of chromospheric emission. The presence of a UV excess in non-accreting pre-main-sequence stars and active stars (when compared to inactive dwarf stars) due to enhanced chromospheric activity is well established (Houdebine et al. 1996; Franchini et al. 1998). In fact, recent studies with Galaxy Evolution Explorer have relied on this excess to identify young stars in nearby star-forming regions (Rodriguez et al. 2011; Shkolnik et al. 2011; Findeisen \& Hillenbrand 2010). This UV excess due to chromospheric emission affects measurements of the excess emission from which accretion luminosities are derived. Several studies have already taken these effects into account. For instance, Valenti et al. (2003) used the average spectrum of several WTTS to estimate the UV excess in low-resolution International Ultraviolet Explorer (IUE) spectra of accreting stars. Similarly, Herczeg \& Hillenbrand (2008) used WTTS templates to measure flux excesses and estimate accretion rates in very low mass objects. However, many determinations of accretion luminosities and accretion rates based on UV fluxes have used dwarf standards as templates (Gullbring et al. 2000; Calvet et al. 2004). The omission of the chromospheric fluxes makes these determinations, and calibrations of line luminosities derived from them, uncertain at the low end.

In this work we have presented UV fluxes of RECX-1, which to date is the only WTTS spectrum with the signal and resolution needed for use as a chromospheric template. We compare the spectrum of the inactive K5 main-sequence star (HD 154363) with that of RECX-1, as shown in Figure 1. The flux between 2000 and $5000 \AA$ from RECX-1 is $0.05 L_{\odot}$ brighter than that from HD 154363. If this luminosity difference were due to accretion, we would have estimated that RECX-1 is accreting at $\log \dot{M}>-8.5 M_{\odot} \mathrm{yr}^{-1}$, which is typical of a CTTS. This is a lower limit because we have not accounted for any excess emission outside of the range of observations. This clearly demonstrates the effect of an active chromosphere on the UV spectrum and shows that inactive main-sequence standards should not be used to measure small NUV excesses. It can be argued that the chromospheric level of RECX-1 is not typical of WTTS because it is a binary. However, with a binary separation > 15 AU (Köhler \& Petr-Gotzens 2002; Brandeker et al. 2006) it is unlikely that the stars are interacting. In any event, a survey of NUV spectra of WTTS of different spectral types and luminosities is needed to determine the characteristic range of chromospheric NUV emission in these stars.

\subsection{Estimate of the Mass Accretion Rate from NUV Emission}

With evidence that RECX-11 is accreting, we use shock models to estimate upper limits to the accretion luminosity and mass accretion rate of RECX-11. A full description of the accretion shock models can be found in Calvet \& Gullbring (1998); here we review the main characteristics. An accretion shock forms at the stellar surface when material from the disk falls onto the star along magnetic field lines (Section 1). The shock formed at the base of the accretion column slows the material to match the stationary photosphere, converting the kinetic energy into thermal energy, and causing the temperature to increase sharply. The shock emits soft $\mathrm{X}$-rays into the pre-shock and post-shock regions and the photosphere below the shock. The heated gas emits the observed excess continuum emission which is strongest in the UV (Calvet \& Gullbring 1998). The accretion shock model is used to fit the NUV excess emission and account for shock emission outside of the range of observations. The model yields the accretion luminosity, from which the mass accretion rate can be derived with knowledge of the stellar mass and radius, through $L_{\text {acc }}=G M \dot{M} / R$. The accretion shock model assumes that all the excess flux in the UV comes from accretion, neglecting any contribution from the outflow. Since the luminosity of the wind is $<10 \%$ of the accretion luminosity for typical mass-loss rates (Cranmer 2009), this assumption is justified.

We model the spectrum of RECX-11 as the sum of the WTTS RECX-1, used as a proxy for the intrinsic stellar emission, and the accretion shock emission; our fit to the observations is shown in Figure 8 and has a $\chi_{\text {red }}^{2} \sim 11.5$. Since the NUV fluxes of RECX-11 are essentially chromospheric, our aim is to determine the amount of shock emission that may be hidden in the spectrum of RECX-11. We find that $\dot{M} \sim 3 \times 10^{-10} M_{\odot} \mathrm{yr}^{-1}$ is the highest value of the mass accretion rate that RECX-11 could have and still not show an excess over the chromospheric emission or veiling in the optical absorption lines. 


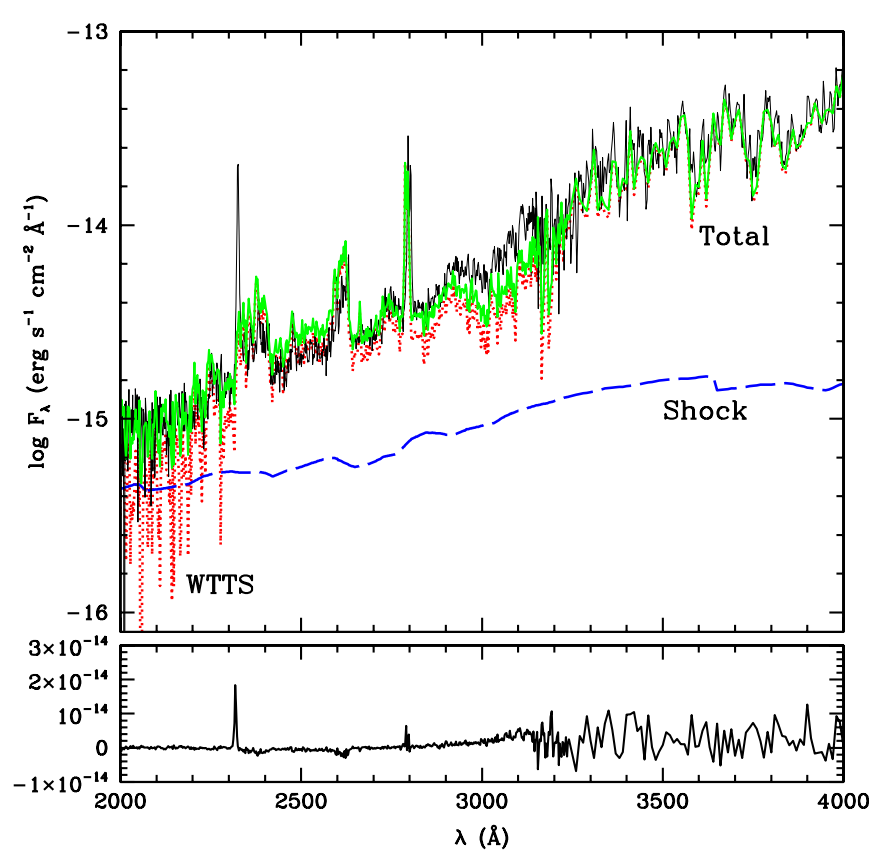

Figure 8. STIS spectrum of RECX-11 compared to predictions of the accretion shock model. Top panel: we show the STIS spectrum of RECX-11 (thin solid line) along with the template NUV and optical emission, the WTTS RECX-1 (red dotted line), and the emission predicted from the accretion shock model (blue long dashed line), with $\dot{M} \leqslant 3 \times 10^{-10} M_{\odot} \mathrm{yr}^{-1}$. The thick solid green spectrum is the accretion shock model added to the WTTS spectrum. Bottom panel: the model (green) was subtracted from the observations (black) and the residuals are shown.

(A color version of this figure is available in the online journal.)

The upper limit on $\dot{M}$ is consistent with estimates of accretion from the emission lines shown in Figures 2 and 4. From the width of the $\mathrm{H} \alpha$ line at $10 \%$ of the maximum and the relation between the $10 \%$ width and $\dot{M}$ of Natta et al. (2004), we find $\dot{M}=2.3 \times 10^{-10} M_{\odot} \mathrm{yr}^{-1}$. Also, from the flux in the C IV $\lambda 1549$ line, we find $M=1.6 \times 10^{-10} M_{\odot} \mathrm{yr}^{-1}$ from the relation between C IV flux and $\dot{M}$ in Valenti et al. (2003). Lawson et al. (2004) fit their $\mathrm{H} \alpha$ line profile of RECX-11 with a magnetospheric accretion model, finding good agreement for a model with a mass accretion rate of $\dot{M}=4 \times 10^{-11} M_{\odot} \mathrm{yr}^{-1}$, also consistent with our upper limit.

\section{DISCUSSION}

\section{1. $L_{\text {acc }}$ versus $L_{U}$}

While it is ideal to measure $L_{\text {acc }}$ from the peak of the excess emission, it is often not possible to obtain these observations in the UV. To overcome this difficulty, Gullbring et al. (1998) calibrated the excess luminosity in the $U$ broadband, $L_{U}$, in terms of the accretion luminosity, $L_{\text {acc }}$, for a sample of stars for which they had medium resolution spectra from 3200 to $5400 \AA$. Accretion luminosities were measured for each star in this sample by extracting the excess flux through veiling measurements and using a slab model to account for energy outside the observed bands. The excess luminosity in $U$ was measured by subtracting the flux in the $U$ band of a mainsequence standard with the same $V$ magnitude from the observed $U$-band fluxes. This correlation was later reproduced using the accretion shock model described in Section 4.2 (Calvet \& Gullbring 1998). The $L_{\text {acc }}$ versus $L_{U}$ relation is commonly utilized in studies of large populations because it provides a convenient method for finding $L_{\text {acc }}$ (Rigliaco et al. 2011;
Ingleby et al. 2009; Grosso et al. 2007; Robberto et al. 2004; White \& Ghez 2001; Rebull et al. 2000; Hartmann et al. 1998). However, as discussed above, the calibration was developed using dwarf stars as templates against which the $U$-band excess was measured and, as we have shown, T Tauri stars have active chromospheres which contribute to the $U$ excess.

The UV excess observed in the WTTS RECX-1 significantly decreases the estimated excess from the accretion shock emission in the NUV. Assuming that the chromospheric emission in RECX-1 is typical for $\sim \mathrm{K} 5$ stars, then the intrinsic chromospheric NUV flux would be $0.05 L_{\odot}$ (Section 4.1). If this NUV excess were interpreted as an "accretion luminosity," using the Gullbring et al. (1998) relation between $L_{\text {acc }}$ and $U$-band excess, the excess luminosity in the $U$ band just due to chromospheric emission would be $0.01 L_{\odot}$. This suggests that for the $\sim$ K5 spectral range, $U$-band excesses and derived accretion luminosities below these limits should be taken with caution. An extensive survey of the intrinsic chromospheric emission in WTTS covering a wide spectral range is necessary to quantify the expected chromospheric emission in CTTS. A re-calibration of the $L_{U}$ versus $L_{\text {acc }}$ relation using characteristic chromospheric contributions may extend its range of application to low values of $L_{\text {acc }}$ and $\dot{M}$.

\subsection{RECX-11 and Disk Evolution}

RECX-11 is in the $\eta$ Chamaeleontis group, with an estimated age of 5-9 Myr. This age range is interesting because disk frequency studies indicate that $\sim 80 \%$ of the original disks have already dissipated by 5 Myr (Hernández et al. 2008). The disk around RECX-11 is then one of the few survivors and a study of its properties can give insight into the mechanisms of disk survival and dissipation.

In the age range of RECX-11, viscous disk evolution would predict a value for $\dot{M}$ between $10^{-10} M_{\odot} \mathrm{yr}^{-1}$ and $10^{-8} M_{\odot} \mathrm{yr}^{-1}$, extrapolating for the dispersion observed in other populations (Calvet et al. 2005). The $M$ for RECX-11 is below this range, so additional disk dispersal mechanisms like photoevaporation may be at play. According to one type of photoevaporation model, EUV photons would ionize the disk surface; a wind is established outside a radius $r_{g}$ where the gas escape velocity becomes higher than the sound speed. The predicted mass-loss rate for EUV photoevaporation is $\sim 10^{-10} M_{\odot} \mathrm{yr}^{-1}$ (Alexander \& Armitage 2007). After the mass accretion rate becomes comparable to the mass-loss rate, mass does not reach the inner disk, which drains onto the star in timescales comparable to the local viscous time, $\sim 10^{5}$ years (Clarke et al. 2001). An inner disk hole is thus created, and EUV irradiation of its edge is expected to quickly erode the rest of the disk (Alexander et al. 2006). For the mass of RECX-11 (Table 1), the value of $r_{g}$ (Clarke et al. 2001) would be $\sim 7$ AU. With further refinements of the theory, the value of the critical radius can be decreased to $\sim 0.1-0.2 r_{g}$ (Font et al. 2004; Adams et al. 2004). This implies that if EUV photoevaporation were active with mass-loss rates greater than the low accretion rate of RECX-11, a hole of at least $0.7 \mathrm{AU}$ should have developed in the RECX-11 disk.

A second type of photoevaporation model includes the effects of stellar X-ray and FUV emission and predicts higher massloss rates than the EUV models (Ercolano et al. 2008; Gorti \& Hollenbach 2009). According to the predictions of the $\mathrm{X}$-ray-driven photoevaporation models, the wind mass-loss rate scales with the X-ray luminosity. The X-ray luminosity of RECX-11 has been recently measured by XMM-Newton to be $3 \times 10^{30} \mathrm{erg} \mathrm{s}^{-1}$ (López-Santiago et al. 2010), very similar to 


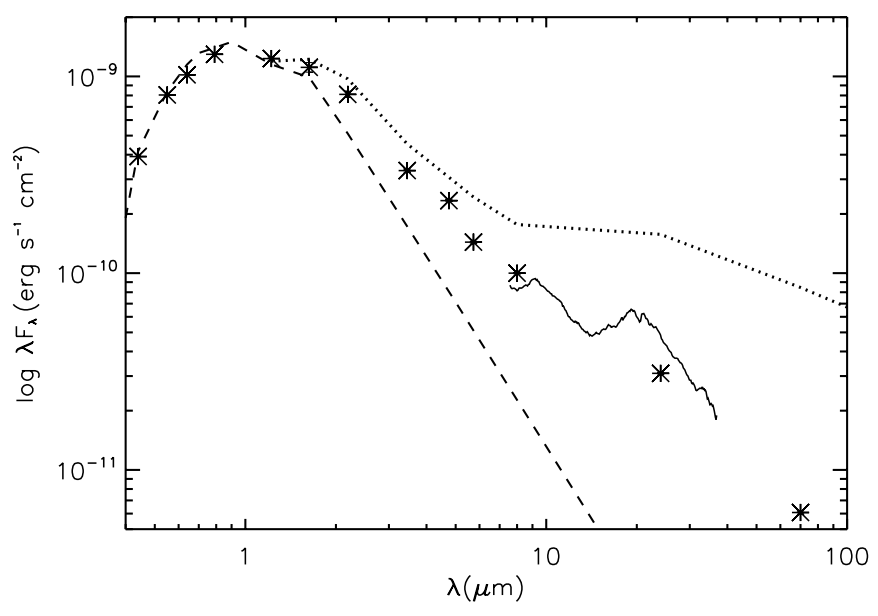

Figure 9. IR spectrum of RECX-11. The IRS spectrum (solid line) and Spitzer photometry (asterisks) show that the IR fluxes are lower than the median IR fluxes of Taurus (dotted line), yet still in excess over the photosphere (dashed line) even in the near-IR. A near-IR excess is due to dust in the inner circumstellar disk, extending all the way to the dust sublimation radius.

the value $1.6 \times 10^{30} \mathrm{erg} \mathrm{s}^{-1}$ estimated from ROSAT observations (Mamajek et al. 2000). The disk of RECX-11 must have been subject to this influx of high-energy radiation for most of its lifetime, since the X-ray luminosity stays approximately constant in the 1-10 Myr age range (Ingleby et al. 2011). With the observed value of the X-ray luminosity, the mass-loss rate predicted by X-ray-driven photoevaporation is $\sim 10^{-8} M_{\odot} \mathrm{yr}^{-1}$, and the low value of the accretion rate would imply that the disk was quickly clearing its inner regions, with timescales $\leqslant 10^{5} \mathrm{yr}$ (Owen et al. 2011). So, for the X-ray photoevaporation models, the innermost disk regions should be cleared or in the process of being cleared.

To find evidence of the effects of photoevaporation on the disk of RECX-11, we have constructed the spectral energy distribution (SED) shown in Figure 9. We plot fluxes at the $B, V$, $R$, and $I_{C}$ bands (Lyo et al. 2004; Lawson et al. 2001) and $J, H$, and $K$ bands from the Two Micron All Sky Survey (Skrutskie et al. 2006) along with Spitzer/IRAC [3.6], [4.5], [5.8], and [8] and MIPS [24] and [70] bands from Sicilia-Aguilar et al. (2009). We also include the Spitzer IRS spectrum, observed in the SL1 and LL modes; the spectrum was downloaded from the Spitzer archive and reduced with version S18.7 of the Spitzer Science Center pipeline using the SMART data reduction package (Higdon et al. 2004) following the description in McClure et al. (2010). The SED of a K5 star, scaled at $J$, is shown for comparison; this SED was constructed with colors taken from Kenyon \& Hartmann (1995). The median SED of Taurus (D'Alessio et al. 1999), also scaled at $J$, is also shown.

The SED of RECX-11 shown in Figure 9 shows excess above the photosphere at all wavelengths $>1.5 \mu \mathrm{m}$. Beyond the nearinfrared, the fluxes are lower than the median SED of Taurus, a proxy for optically thick disks in which dust is present all the way into the dust sublimation radius. The slope of the SED between 13 and $31 \mu \mathrm{m}$ of $n_{13-31}=-0.8$ is steeper than the median value of -0.4 for Taurus, indicating a significant degree of dust settling in the RECX-11 disk (Furlan et al. 2006, 2009; McClure et al. 2010). In addition, the profiles of the $10 \mu \mathrm{m}$ and $20 \mu \mathrm{m}$ silicate features in the IRS spectrum are wider than those found in the interstellar medium (Sicilia-Aguilar et al. 2009) and contain substructure consistent with significant dust evolution, either in the form of grain growth or crystallization (Watson et al. 2009).

Despite the high degree of evolution of dust in the disk, the SED of RECX-11 does not show indications of an inner cleared region. In fact, the location of RECX-11 in the K-[8] versus $\mathrm{K}-$ [24] diagram corresponds to that of full disks according to the study of circumstellar disks at various stages of evolution by Ercolano et al. (2011). Moreover, the SED of RECX-11 shows a substantial near-IR excess, comparable to the median SED of Taurus, despite the low fluxes at longer wavelengths. The largest contributor to the near-IR excess in T Tauri star disks is the optically thick "wall" at the dust destruction radius, the sharp transition between the dust and gas disk (D'Alessio et al. 2006; Dullemond \& Monnier 2010). For the parameters of RECX-11, the dust destruction radius is of the order of $\sim 0.06 \mathrm{AU}$, taking the simple expression for the equilibrium temperature, with $T=1400 \mathrm{~K}$ as the dust sublimation temperature and $T_{\text {eff }}=$ $4350 \mathrm{~K}$ for a K5 star (Dullemond \& Monnier 2010).

The evidence then seems to rule out inner clearing in the disk of RECX-11. First, it is accreting, so mass has to reach the magnetospheric radius, 3-5 stellar radii, $\sim 0.03$ AU. Substantial $\mathrm{H}_{2}$ emission is observed, coming from gas regions as close in as $0.01-0.1 \mathrm{AU}$ to the central star, typical of all accretors. Moreover, dust extends inward to $\sim 0.06 \mathrm{AU}$. These inner radii, where disk material is present, are not consistent with those predicted for photoevaporation models, according to which the innermost $<0.1 \mathrm{AU}$ of the disk should be clear. It could be argued that the disk of RECX-11 has been caught in the short phase when the inner disk is still accreting its remaining mass onto the star. However, the recent analysis of the disk of another star in the $\eta$ Cha group, ET Cha (ECHA J0843.3-7915 = RECX-15), casts some doubt on this suggestion (Woitke et al. 2011). The disk of ET Cha is also accreting (Lawson et al. 2004); it is very evolved with very low dust and gas masses, but still has a very substantial near-IR excess consistent with emission from the dust destruction radius (Woitke et al. 2011). For a 5-9 Myr population of less than 20 stars (Mamajek et al. 2000) the probability of finding two stars in a phase lasting only $\sim 10^{5}$ years is low. Overall, the evidence provided by low accretors in evolved populations suggests that current models of disk dissipation by photoevaporation with high mass-loss rates may need to be revisited. Our upper limit on $\dot{M}$ does not, however, rule out photoevaporation at the low mass-loss rates predicted by the EUV photoevaporation models.

\section{SUMMARY AND CONCLUSIONS}

We used multi-wavelength observations of the CTTS RECX-11 and the WTTS RECX-1 to investigate their accretion properties. We found the following.

1. Multi-wavelength observations provide the clearest picture of the accretion properties of a low- $\dot{M}$ T Tauri star. Observations of molecular gas in the FUV may be the most sensitive accretion probe, since these lines are only present in accreting sources (Ingleby et al. 2009), and their line profiles are consistent with an origin in the disk, very close to the star. In the optical and near-infrared, the most sensitive indications of accretion come from high-resolution observations of the $\mathrm{H} \alpha$ and $\mathrm{He}$ I $\lambda 10830$ emission lines. These show variable and multiple redshifted absorption components which reveal the complex structure of the magnetosphere in a low- $\dot{M}$ source. For very low values of $\dot{M}$, lines normally used as accretion indicators, 
such as the Ca II IR triplet lines, arise mostly in the stellar chromosphere and are not sensitive to accretion.

2. The contribution from the stellar chromosphere to the NUV flux can be substantial and may result in estimates of the accretion luminosity from $U$ excesses that are significantly higher than the actual value of $L_{\text {acc }}$. For RECX-1, a K5 star that is not accreting, as determined from its narrow $\mathrm{H} \alpha$ line profile and from the lack of $\mathrm{H}_{2}$ lines in the FUV, its chromospheric NUV excess over the photosphere of a standard dwarf star would result in incorrect estimates of $L_{\text {acc }} \sim 0.05 L_{\odot}$ and $\dot{M} \sim 3 \times 10^{-9} M_{\odot} \mathrm{yr}^{-1}$. For the middle $\mathrm{K}$ spectral range, estimates of accretion less than these limits based on methods calibrated with standard dwarf stars should be taken with caution.

3. When accounting for the active chromospheres of $\mathrm{T}$ Tauri stars by using a WTTS as a template against which to measure the NUV excess, we determine a very low $\dot{M}$ for RECX-11, $\leqslant 3 \times 10^{-10} M_{\odot} \mathrm{yr}^{-1}$.

4. Given the low mass accretion rate, the high X-ray luminosity, and the advanced age of RECX-11, photoevaporation is expected to be driving the final evolution of its disk; if this was the case, the innermost disk regions, $\leqslant 0.1 \mathrm{AU}$, should be clear. However, we find evidence for gas and dust in the innermost disk of RECX-11, as indicated by ongoing accretion, the detection of $\mathrm{H}_{2}$ lines formed in the innermost disk, and emission from optically thick dust at the dust destruction radius at levels consistent with other CTTS. This, together with similar evidence provided by other slowly accreting, old disks, suggests that models for photoevaporation-driven disk dissipation resulting in high values of the mass-loss rate should be revisited.

We thank Will Fischer for help reducing the Phoenix data to obtain the He I line profile. We thank the SMARTS service for obtaining the SMARTS spectra. Stony Brook University is a member of the SMARTS partnership. This work was supported by NASA grants for Guest Observer program 11616 to the University of Michigan, Caltech, Stony Brook University, and the University of Colorado. Based on observations made with the NASA/ESA Hubble Space Telescope, obtained from the Space Telescope Science Institute data archive. STScI is operated by the Association of Universities for Research in Astronomy, Inc. under NASA contract NAS 5-26555. C.E. was supported by the National Science Foundation under award no. 0901947. R.A. acknowledges support from the Science \& Technology Facilities Council (STFC) through an Advanced Fellowship (ST/G00711X/1).

\section{REFERENCES}

Adams, F. C., Hollenbach, D., Laughlin, G., \& Gorti, U. 2004, ApJ, 611, 360 Alexander, R. D., \& Armitage, P. J. 2007, MNRAS, 375, 500

Alexander, R. D., Clarke, C. J., \& Pringle, J. E. 2006, MNRAS, 369, 229

Ardila, D. R., \& Johns-Krull, C. M. 2009, in AIP Conf. Proc. 1094, Cool Stars, Stellar Systems and the Sun, ed. E. Stempels (Melville, NY: AIP), 309

Bary, J. S., Weintraub, D. A., Shukla, S. J., Leisenring, J. M., \& Kastner, J. H. 2008, ApJ, 678, 1088

Bergin, E., Calvet, N., Sitko, M. L., et al. 2004, ApJ, 614, L133

Bernstein, R., Shectman, S. A., Gunnels, S. M., Mochnacki, S., \& Athey, A. E. 2003, Proc. SPIE, 4841, 1694

Bethell, T., \& Bergin, E. 2009, Science, 326, 1675

Bitner, M. A., Richter, M. J., Lacy, J. H., et al. 2008, ApJ, 688, 1326

Bouvier, J., Alencar, S. H. P., Boutelier, T., et al. 2007, A\&A, 463, 1017

Brandeker, A., Jayawardhana, R., Khavari, P., Haisch, K. E., Jr., \& Mardones, D. 2006, ApJ, 652, 1572

Calvet, N., \& Gullbring, E. 1998, ApJ, 509, 802
Calvet, N., Briceño, C., Hernández, J., et al. 2005, AJ, 129, 935

Calvet, N., Muzerolle, J., Briceño, C., et al. 2004, AJ, 128, 1294

Cardini, D., \& Cassatella, A. 2007, ApJ, 666, 393

Carr, J. S., \& Najita, J. R. 2008, Science, 319, 1504

Clarke, C. J., Gendrin, A., \& Sotomayor, M. 2001, MNRAS, 328, 485

Cranmer, S. R. 2009, ApJ, 706, 824

D’Alessio, P., Calvet, N., Hartmann, L., Franco-Hernández, R., \& Servín, H 2006, ApJ, 638, 314

D’Alessio, P., Calvet, N., Hartmann, L., Lizano, S., \& Cantó, J. 1999, ApJ, 527, 893

Danforth, C. W., Keeney, B. A., Stocke, J. T., Shull, J. M., \& Yao, Y. 2010, ApJ, 720, 976

Dullemond, C. P., \& Monnier, J. D. 2010, ARA\&A, 48, 205

Edwards, S., Fischer, W., Hillenbrand, L., \& Kwan, J. 2006, ApJ, 646, 319

Ercolano, B., Clarke, C. J., \& Hall, A. C. 2011, MNRAS, 410, 671

Ercolano, B., Drake, J. J., Raymond, J. C., \& Clarke, C. C. 2008, ApJ, 688, 398

Findeisen, K., \& Hillenbrand, L. 2010, AJ, 139, 1338

Fischer, W., Kwan, J., Edwards, S., \& Hillenbrand, L. 2008, ApJ, 687, 1117

Font, A. S., McCarthy, I. G., Johnstone, D., \& Ballantyne, D. R. 2004, ApJ, 607,890

France, K., Linsky, J. L., Brown, A., Froning, C. S., \& Béland, S. 2010, ApJ, 715,596

France, K., Schindhelm, E., Burgh, E. B., et al. 2011, ApJ, 734, 31

Franchini, M., Morossi, C., \& Malagnini, M. L. 1998, ApJ, 508, 370

Furlan, E., Hartmann, L., Calvet, N., et al. 2006, ApJS, 165, 568

Furlan, E., Watson, D. M., McClure, M. K., et al. 2009, ApJ, 703, 1964

Gómez de Castro, A. I., \& Ferro-Fontán, C. 2005, MNRAS, 362, 569

Gorti, U., Dullemond, C. P., \& Hollenbach, D. 2009, ApJ, 705, 1237

Gorti, U., \& Hollenbach, D. 2009, ApJ, 690, 1539

Grosso, N., Audard, M., Bouvier, J., Briggs, K. R., \& Güdel, M. 2007, A\&A, 468,557

Gullbring, E., Calvet, N., Muzerolle, J., \& Hartmann, L. 2000, ApJ, 544, 927

Gullbring, E., Hartmann, L., Briceno, C., \& Calvet, N. 1998, ApJ, 492, 323

Günther, H. M., Schmitt, J. H. M. M., Robrade, J., \& Liefke, C. 2007, A\&A, 466, 1111

Hartigan, P., Edwards, S., \& Ghandour, L. 1995, ApJ, 452, 736

Hartmann, L., Calvet, N., Gullbring, E., \& D’Alessio, P. 1998, ApJ, 495, 385

Heap, S. R., \& Lindler, D. J. 2007, in ASP Conf. Ser. 374, From Stars to Galaxies: Building the Pieces to Build Up the Universe, ed. A. Vallenari, R. Tantalo, L. Portinari, \& A. Moretti (San Francisco, CA: ASP), 409

Herczeg, G. J., \& Hillenbrand, L. A. 2008, ApJ, 681, 594

Herczeg, G. J., Linsky, J. L., Valenti, J. A., Johns-Krull, C. M., \& Wood, B. E. 2002, ApJ, 572, 310

Herczeg, G. J., Wood, B. E., Linsky, J. L., Valenti, J. A., \& Johns-Krull, C. M. 2004, ApJ, 607, 369

Hernández, J., Hartmann, L., Calvet, N., et al. 2008, ApJ, 686, 1195

Higdon, S. J. U., Devost, D., Higdon, J. L., et al. 2004, PASP, 116, 975

Holmberg, J., Nordström, B., \& Andersen, J. 2009, A\&A, 501, 941

Houdebine, E. R., Mathioudakis, M., Doyle, J. G., \& Foing, B. H. 1996, A\&A, 305,209

Ingleby, L., Calvet, N., Bergin, E., et al. 2009, ApJ, 703, L137

Ingleby, L., Calvet, N., Hernández, J., et al. 2011, AJ, 141, 127

Jayawardhana, R., Coffey, J., Scholz, A., Brandeker, A., \& van Kerkwijk, M. H. 2006, ApJ, 648, 1206

Johns-Krull, C. M., Valenti, J. A., \& Linsky, J. L. 2000, ApJ, 539, 815

Kenyon, S. J., \& Hartmann, L. 1995, ApJS, 101, 117

Köhler, R., \& Petr-Gotzens, M. G. 2002, AJ, 124, 2899

Kurosawa, R., Harries, T. J., \& Symington, N. H. 2006, MNRAS, 370, 580

Lawson, W. A., Crause, L. A., Mamajek, E. E., \& Feigelson, E. D. 2001, MNRAS, 321, 57

Lawson, W. A., Lyo, A.-R., \& Muzerolle, J. 2004, MNRAS, 351, L39

Lima, G. H. R. A., Alencar, S. H. P., Calvet, N., Hartmann, L., \& Muzerolle, J. 2010, A\&A, 522, A104

López-Santiago, J., Albacete Colombo, J. F., \& López-García, M. A. 2010, A\&A, 524, A97

Lord, S. D. 1992, NASA Technical Memorandum 103957

Luhman, K. L., \& Steeghs, D. 2004, ApJ, 609, 917

Lyo, A.-R., Lawson, W. A., \& Bessell, M. S. 2004, MNRAS, 355, 363

Mamajek, E. E., Lawson, W. A., \& Feigelson, E. D. 1999, ApJ, 516, L77

Mamajek, E. E., Lawson, W. A., \& Feigelson, E. D. 2000, ApJ, 544, 356

Martínez-Arnáiz, R., Maldonado, J., Montes, D., Eiroa, C., \& Montesinos, B. 2010, A\&A, 520, A79

McClure, M. K., Furlan, E., Manoj, P., et al. 2010, ApJS, 188, 75

Meijerink, R., Glassgold, A. E., \& Najita, J. R. 2008, ApJ, 676, 518

Muzerolle, J., Calvet, N., \& Hartmann, L. 1998, ApJ, 492, 743

Muzerolle, J., Calvet, N., \& Hartmann, L. 2001, ApJ, 550, 944 
Muzerolle, J., Hillenbrand, L., Calvet, N., Briceño, C., \& Hartmann, L. 2003, ApJ, 592, 266

Najita, J. R., Carr, J. S., Strom, S. E., et al. 2010, ApJ, 712, 274

Natta, A., Testi, L., Muzerolle, J., et al. 2004, A\&A, 424, 603

Owen, J. E., Ercolano, B., \& Clarke, C. J. 2011, MNRAS, 412, 13

Pascucci, I., Apai, D., Luhman, K., et al. 2009, ApJ, 696, 143

Pinte, C., Woitke, P., Mnard, F., et al. 2010, A\&A, 518, L126

Rayner, J. T., Cushing, M. C., \& Vacca, W. D. 2009, ApJS, 185, 289

Rebull, L. M., Hillenbrand, L. A., Strom, S. E., et al. 2000, AJ, 119, 3026

Rigliaco, E., Natta, A., Randich, S., Testi, L., \& Biazzo, K. 2011, A\&A, 525, A47

Robberto, M., Song, J., Mora Carrillo, G., et al. 2004, ApJ, 606, 952

Rodriguez, D. R., Bessell, M. S., Zuckerman, B., \& Kastner, J. H. 2011, ApJ, 727, 62

Shkolnik, E. L., Liu, M. C., Reid, I. N., Dupuy, T., \& Weinberger, A. J. 2011, ApJ, 727,6
Sicilia-Aguilar, A., Bouwman, J., Juhsz, A., et al. 2009, ApJ, 701, 1188

Siess, L., Dufour, E., \& Forestini, M. 2000, A\&A, 358, 593

Skrutskie, M. F., Cutri, R. M., Stiening, R., et al. 2006, AJ, 131, 1163

Takeda, G., Ford, E. B., Sills, A., et al. 2007, ApJS, 168, 297

Tody, D. 1993, in ASP Conf. Ser. 52, Astronomical Data Analysis Software and Systems II, ed. R. J. Hanisch, R. J. V. Brissenden, \& J. Barnes (San Francisco, CA: ASP), 173

Valenti, J. A., Fallon, A. A., \& Johns-Krull, C. M. 2003, ApJS, 147, 305

Walter, F. M. 1999, in ASP Conf. Ser. 158, Solar and Stellar Activity: Similarities and Differences, ed. C. J. Butler \& J. G. Doyle (San Francisco, CA: ASP), 87

Watson, D. M., Leisenring, J. M., Furlan, E., et al. 2009, ApJS, 180, 84

White, R. J., \& Basri, G. 2003, ApJ, 582, 1109

White, R. J., \& Ghez, A. M. 2001, ApJ, 556, 265

Woitke, P., Riaz, B., Duchêne, G., et al. 2011, A\&A, 534, A44

Yang, H., Linsky, J. L., \& France, K. 2011, ApJ, 730, L10 